\title{
Least-Squares Finite Element Method for the Steady Upper-Convected Maxwell Fluid
}

\author{
Shaoling Zhou1,2, Lei Hou1 \\ ${ }^{1}$ Department of Mathematics, Shanghai University, Shanghai, China \\ ${ }^{2}$ School of Science, Hebei University of Engineering, Handan, China \\ Email: zhoushaoling@shu.edu.cn, houlei@shu.edu.cn
}

Received 7 March 2015; accepted 26 March 2015; published 3 April 2015

Copyright $@ 2015$ by authors and Scientific Research Publishing Inc.

This work is licensed under the Creative Commons Attribution International License (CC BY). http://creativecommons.org/licenses/by/4.0/

(c) (i) Open Access

\begin{abstract}
In this paper, a least-squares finite element method for the upper-convected Maxell (UCM) fluid is proposed. We first linearize the constitutive and momentum equations and then apply a leastsquares method to the linearized version of the viscoelastic UCM model. The $L^{2}$ least-squares functional involves the residuals of each equation multiplied by proper weights. The corresponding homogeneous functional is equivalent to a natural norm. The error estimates of the finite element solution are analyzed when the conforming piecewise polynomial elements are used for the unknowns.
\end{abstract}

\section{Keywords}

Upper-Convected Maxwell Fluid, Least-Squares Finite Element Method, Viscoelastic Fluid Model

\section{Introduction}

In recent years, there has been an increased interest in the least-squares finite element method for the approximation of partial differential equations, see e.g. [1]-[6]. This technique is attractive because the linear systems generated by the discretization are symmetric and positive definite, thus the algebraic system can be solved by fast direct or iterative algorithms. Moreover, in contrast to the mixed finite element method, the inf-sup or LBB type of conditions is naturally satisfied. However, without the weights in the least-squares functional, this method results in poor numerical solutions even for simple problems. In [7], Bochev and Gunzburger pointed that the weighted least-squares method was optimal for the velocity-pressure-stress formulation of the Stokes equations. The weighted least-squares method has also been used to solve other viscoelastic problems, such as the Oldroyd-B, Carreau, and Phan-Thien-Tanner models [1] [3] [8].

In the viscoelastic fluids of the differential type, the constitutive equations consist of an algebraic tensorial 
relationship between the stress tensor and the rate of deformation tensor. The upper-convected Maxwell fluid [9] is the simplest, if not the easiest, representative of that class and has served as a model fluid for developing numerical techniques. The purpose of this paper is to present a finite element method for the upper-convected Maxwell fluid which is one of the most used viscoelastic models. The nonlinear model is first approached by linearizing the equations and a weighted least-squares finite element method is applied to solve the linear equations. Error estimates of the finite element solutions to the linear system are derived.

\section{Governing Equations}

Assume that $\Omega$ is a bounded and connected domain in $\mathbb{R}^{2}$ with Lipschitz boundary $\Gamma$. We consider the steady incompressible flows governed by the conservation equations for mass and momentum

$$
\begin{aligned}
& \nabla \cdot \boldsymbol{u}=0 \\
& \rho \boldsymbol{u} \cdot \nabla \boldsymbol{u}+\nabla p-\nabla \cdot \boldsymbol{\sigma}=\boldsymbol{f}
\end{aligned}
$$

where $\boldsymbol{u}$ denotes the velocity vector, $\rho$ the constant density, $p$ the pressure, $\boldsymbol{\sigma}$ the extra-stress tensor and $f$ the body force.

For the upper-convected Maxwell model, the extra-stress tensor $\sigma$ satisfies the following constitutive equation

$$
\sigma+\lambda \sigma_{(1)}=2 \eta \varepsilon(\boldsymbol{u})
$$

where $\eta$ is the constant viscosity, $\lambda$ the relaxation time and $\varepsilon(\boldsymbol{u})=\left(\nabla \boldsymbol{u}+\nabla \boldsymbol{u}^{\mathrm{T}}\right) / 2$ the standard strain rate tensor. The subscript (1) denotes the upper-convected material derivative

$$
\sigma_{(1)}=\boldsymbol{u} \cdot \nabla \boldsymbol{\sigma}-g(\sigma, \nabla \boldsymbol{u})
$$

and

$$
g(\boldsymbol{\sigma}, \nabla \mathbf{u})=\nabla \mathbf{u} \cdot \boldsymbol{\sigma}+\boldsymbol{\sigma} \cdot \nabla \boldsymbol{u}^{\mathrm{T}} .
$$

To simplify our analysis, homogeneous boundary conditions are assumed on $\Gamma$. The results in this article can be extended to nonhomogeneous boundary conditions easily. Collecting (2.1)-(2.3), we obtain the steady UCM model

$$
\begin{cases}\nabla \cdot \boldsymbol{u}=0, & \text { in } \Omega, \\ \rho \boldsymbol{u} \cdot \nabla \boldsymbol{u}+\nabla p-\nabla \cdot \boldsymbol{\sigma}=f, & \text { in } \Omega, \\ \boldsymbol{\sigma}+\lambda(\boldsymbol{u} \cdot \nabla \boldsymbol{\sigma}-g(\boldsymbol{\sigma}, \nabla \boldsymbol{u}))=2 \eta \varepsilon(\boldsymbol{u}), & \text { in } \Omega, \\ \boldsymbol{u}=0, & \text { on } \Gamma .\end{cases}
$$

\section{Mathematical Notation and Preliminaries}

Throughout the paper, we use the standard notation and definition for the Sobolev spaces $H^{s}(\Omega), s \geq 0$, with inner products and norms denoted by $(\cdot, \cdot)_{s}$ and \|\|$_{s}$. For $s=0$, we write $L^{2}(\Omega),(\cdot, \cdot)$ and $\|\cdot\|$. As usual, $H_{0}^{s}(\Omega)$ denotes the closure of $C_{0}^{\infty}(\Omega)$ with respect to the norm $\|\cdot\|_{s}$ and $L_{0}^{2}(\Omega)$ denotes the space of square integrable functions with zero mean

$$
L_{0}^{2}(\Omega) \boxminus\left\{p \in L^{2}(\Omega): \int_{\Omega} p \mathrm{~d} x=0\right\} .
$$

The spaces $H^{-s}(\Omega)$ with positive values of $s$ is defined as the dual space of $H_{0}^{s}(\Omega)$ with the following norm

$$
\|\phi\|_{-s}=\sup _{0 \neq \psi \in H_{0}^{s}} \frac{\langle\phi, \psi\rangle}{\|\psi\|_{s}}
$$

where $\langle\cdot, \cdot\rangle$ stands for the duality pairing between $H^{-s}(\Omega)$ and $H_{0}^{s}(\Omega)$.

We use the following approximation

$$
\mathbf{u}_{1} \approx \boldsymbol{u}, \quad \sigma_{1} \approx \sigma
$$


to linearize the equations in (2.4). Moreover we assume that $\nabla \cdot \boldsymbol{u}_{1}=0$ and the approximation satisfies

$$
\max \left\{\left\|\mathbf{u}_{1}\right\|_{\infty},\left\|\nabla \mathbf{u}_{1}\right\|_{\infty},\left\|\boldsymbol{\sigma}_{1}\right\|_{\infty},\left\|\nabla \boldsymbol{\sigma}_{1}\right\|_{\infty}\right\} \leq M<\infty .
$$

We introduce the replacement rules

$$
\begin{aligned}
& \boldsymbol{u} \cdot \nabla \boldsymbol{u} \approx \boldsymbol{u} \cdot \nabla \boldsymbol{u}_{1}+\boldsymbol{u}_{1} \cdot \nabla \boldsymbol{u}-\boldsymbol{u}_{1} \cdot \nabla \boldsymbol{u}_{1} \\
& \boldsymbol{u} \cdot \nabla \boldsymbol{\sigma} \approx \boldsymbol{u} \cdot \nabla \boldsymbol{\sigma}_{1}+\boldsymbol{u}_{1} \cdot \nabla \boldsymbol{\sigma}-\boldsymbol{u}_{1} \cdot \nabla \boldsymbol{\sigma}_{1} \\
& g(\boldsymbol{\sigma}, \nabla \boldsymbol{u}) \approx g\left(\boldsymbol{\sigma}_{1}, \nabla \boldsymbol{u}\right)+g\left(\boldsymbol{\sigma}, \nabla \boldsymbol{u}_{1}\right)-g\left(\boldsymbol{\sigma}_{1}, \nabla \mathbf{u}_{1}\right)
\end{aligned}
$$

which result in the linearized system

$$
\begin{cases}\nabla \cdot \boldsymbol{u}=0, & \text { in } \Omega, \\ \rho\left(\boldsymbol{u} \cdot \nabla \mathbf{u}_{1}+\boldsymbol{u}_{1} \cdot \nabla \boldsymbol{u}\right)+\nabla p-\nabla \cdot \boldsymbol{\sigma}=\hat{\boldsymbol{f}} & \text { in } \Omega, \\ \boldsymbol{\sigma}+\lambda\left(\boldsymbol{u}_{1} \cdot \nabla \boldsymbol{\sigma}+B(\boldsymbol{u}, \boldsymbol{\sigma})\right)-2 \eta \varepsilon(\boldsymbol{u})=\boldsymbol{F}, & \text { in } \Omega, \\ \boldsymbol{u}=0, & \text { on } \Gamma .\end{cases}
$$

where

$$
\begin{aligned}
& \hat{\boldsymbol{f}}=\boldsymbol{f}+\rho \boldsymbol{u}_{1} \cdot \nabla \mathbf{u}_{1} \\
& B(\boldsymbol{u}, \boldsymbol{\sigma})=\lambda\left(\boldsymbol{u} \cdot \nabla \boldsymbol{\sigma}_{1}-g\left(\boldsymbol{\sigma}_{1}, \nabla \boldsymbol{u}\right)-g\left(\boldsymbol{\sigma}, \nabla \mathbf{u}_{1}\right)\right) \\
& \boldsymbol{F}=\lambda\left(\boldsymbol{u}_{1} \cdot \nabla \boldsymbol{\sigma}_{1}+g\left(\boldsymbol{\sigma}_{1}, \nabla \boldsymbol{u}_{1}\right)\right)
\end{aligned}
$$

The velocity $\boldsymbol{u}$, the pressure $p$ and the extra-stress tensor $\sigma$ belong to their respective spaces

$$
\begin{aligned}
& V=H_{0}^{1}(\Omega)=\left\{v \in H^{1}(\Omega):\left.v\right|_{\Gamma}=0\right\} \\
& Q=L_{0}^{2}(\Omega)=\left\{q \in L^{2}(\Omega): \int_{\Omega} p \mathrm{~d} x=0\right\} \\
& \Sigma=\left\{\tau=\left(\tau_{i j}\right): \tau_{i j}=\tau_{j i}, \tau_{i j} \in L^{2}(\Omega)\right\}
\end{aligned}
$$

and let $X=V \times Q \times \Sigma$.

Based on [7], we define the weighted least-squares functional for the linearized system (3.3)

$$
\begin{aligned}
J(\boldsymbol{u}, p, \boldsymbol{\sigma} ; \hat{\boldsymbol{f}}, \boldsymbol{F}) & =\|\nabla \cdot \boldsymbol{u}\|^{2}+\left\|\rho\left(\boldsymbol{u} \cdot \nabla \boldsymbol{u}_{1}+\boldsymbol{u}_{1} \cdot \nabla \boldsymbol{u}\right)+\nabla p-\nabla \cdot \boldsymbol{\sigma}-\hat{\boldsymbol{f}}\right\|_{-1}^{2} \\
& +\left\|\boldsymbol{\sigma}+\lambda\left(\boldsymbol{u}_{1} \cdot \nabla \boldsymbol{\sigma}+B(\boldsymbol{u}, \boldsymbol{\sigma})\right)-2 \eta \varepsilon(\boldsymbol{u})-\boldsymbol{F}\right\|^{2}
\end{aligned}
$$

Now we show that the homogeneous least-squares functional of (3.4) is equivalent to the norm

$$
\|(\boldsymbol{u}, p, \boldsymbol{\sigma})\|=\|\boldsymbol{u}\|_{1}^{2}+\|p\|^{2}+\|\boldsymbol{\sigma}\|^{2}+\left\|\boldsymbol{u}_{1} \cdot \nabla \boldsymbol{\sigma}\right\|^{2}
$$

Theorem 1. There exist positive constants $c_{1}$ and $c_{2}$, which depend on $\rho, M$ and $\eta$, such that

$$
c_{1}\|(\boldsymbol{u}, p, \boldsymbol{\sigma})\|^{2} \leq J(\boldsymbol{u}, p, \boldsymbol{\sigma} ; \mathbf{0}, \mathbf{0}) \leq c_{2}\|(\boldsymbol{u}, p, \boldsymbol{\sigma})\|^{2}
$$

hold for any $(\boldsymbol{u}, p, \boldsymbol{\sigma}) \in X$.

Proof. The upper bound in (3.5) follows easily from the triangle inequality and (3.2). For the lower bound, we will show that

where

$$
\|(\mathbf{u}, p, \boldsymbol{\sigma})\|^{2} \leq C \Psi
$$

$$
\Psi=\|\nabla \cdot \boldsymbol{u}\|^{2}+\left\|\boldsymbol{\sigma}+\lambda \mathbf{u}_{1} \cdot \nabla \boldsymbol{\sigma}-2 \eta \varepsilon(\boldsymbol{u})\right\|^{2}+\left\|\rho\left(\boldsymbol{u} \cdot \nabla \mathbf{u}_{1}+\boldsymbol{u}_{1} \cdot \nabla \boldsymbol{u}\right)+\nabla p-\nabla \cdot \sigma\right\|_{-1}^{2} .
$$

Using the Green's formula and Cauchy-Schwarz inequality, we obtain 


$$
\begin{aligned}
\langle\nabla p, \varphi\rangle & =\left\langle\rho\left(\boldsymbol{u} \cdot \nabla \mathbf{u}_{1}+\boldsymbol{u}_{1} \cdot \nabla \boldsymbol{u}\right)+\nabla p-\nabla \cdot \boldsymbol{\sigma}, \varphi\right\rangle-\langle\boldsymbol{\sigma}, \nabla \varphi\rangle-\rho\left\langle\boldsymbol{u} \cdot \nabla \boldsymbol{u}_{1}+\boldsymbol{u}_{1} \cdot \nabla \boldsymbol{u}, \varphi\right\rangle \\
& \leq C\left(\left\|\left(\boldsymbol{u} \cdot \nabla \boldsymbol{u}_{1}+\boldsymbol{u}_{1} \cdot \nabla \boldsymbol{u}\right)+\nabla p-\nabla \cdot \boldsymbol{\sigma}\right\|_{-1}+\|\boldsymbol{\sigma}\|+\|\boldsymbol{u}\|_{1}\right),
\end{aligned}
$$

for any $\varphi \in H_{0}^{1}(\Omega)$. By using Lemma 2.1 in [10] as

we obtain

$$
\|p\| \leq C\|\nabla p\|_{-1}
$$

$$
\|p\| \leq C\|\nabla p\|_{-1} \leq C\left(\left\|\left(\boldsymbol{u} \cdot \nabla \boldsymbol{u}_{1}+\boldsymbol{u}_{1} \cdot \nabla \boldsymbol{u}\right)+\nabla p-\nabla \cdot \boldsymbol{\sigma}\right\|_{-1}+\|\boldsymbol{\sigma}\|+\|\boldsymbol{u}\|_{1}\right)
$$

which implies that

$$
\|p\| \leq C\left(\Psi^{1 / 2}+\|\sigma\|+\|\boldsymbol{u}\|_{1}\right) .
$$

Similarly, we have

$$
\begin{aligned}
\lambda\left\|\boldsymbol{u}_{1} \cdot \nabla \boldsymbol{\sigma}\right\| & \leq\left\|\boldsymbol{\sigma}+\lambda \boldsymbol{u}_{1} \cdot \nabla \boldsymbol{\sigma}-2 \eta \varepsilon(\boldsymbol{u})\right\|+\|\boldsymbol{\sigma}\|+2 \eta\|\varepsilon(\boldsymbol{u})\| \\
& \leq C\left(\Psi^{1 / 2}+\|\boldsymbol{\sigma}\|+\|\boldsymbol{u}\|_{1}\right) .
\end{aligned}
$$

By the arguments similar to Theorem 4.1 in [11], we obtain

$$
\|\sigma\|+\|\boldsymbol{u}\|_{1} \leq C \Psi^{1 / 2} .
$$

Combining (3.7)-(3.9) yields (3.6).

From the inequality $\|\alpha+\beta\|^{2} \geq\|\alpha\|^{2} / 2-\|\beta\|^{2}$, we establish

$$
\begin{aligned}
& J(\boldsymbol{u}, p, \boldsymbol{\sigma} ; \mathbf{0 , 0}) \\
& \geq\|\nabla \cdot \boldsymbol{u}\|^{2}+\left\|\boldsymbol{\sigma}+\lambda \mathbf{u}_{1} \cdot \nabla \boldsymbol{\sigma}-2 \eta \varepsilon(\boldsymbol{u})\right\|^{2} / 2-B(\boldsymbol{u}, \boldsymbol{\sigma})^{2}+\left\|\rho\left(\boldsymbol{u} \cdot \nabla \boldsymbol{u}_{1}+\boldsymbol{u}_{1} \cdot \nabla \boldsymbol{u}\right)+\nabla p-\nabla \cdot \boldsymbol{\sigma}\right\|_{-1}^{2} \\
& \geq\left(\|\nabla \cdot \boldsymbol{u}\|^{2}+\left\|\boldsymbol{\sigma}+\lambda \boldsymbol{u}_{1} \cdot \nabla \boldsymbol{\sigma}-2 \eta \varepsilon(\boldsymbol{u})\right\|^{2}+\left[\rho\left(\boldsymbol{u} \cdot \nabla \mathbf{u}_{1}+\boldsymbol{u}_{1} \cdot \nabla \boldsymbol{u}\right)+\nabla p-\nabla \cdot \boldsymbol{\sigma}\right]_{-1}^{2}\right) / 2-B(\boldsymbol{u}, \boldsymbol{\sigma})^{2} \\
& =\Psi / 2-B(\boldsymbol{u}, \boldsymbol{\sigma})^{2}
\end{aligned}
$$

Note that

$$
\|B(\boldsymbol{u}, \boldsymbol{\sigma})\|^{2}=\lambda^{2}\left\|\boldsymbol{u} \cdot \nabla \boldsymbol{\sigma}_{1}-g\left(\boldsymbol{\sigma}_{1}, \nabla \mathbf{u}\right)-g\left(\boldsymbol{\sigma}, \nabla \boldsymbol{u}_{1}\right)\right\|^{2} \leq C \lambda^{2} M^{2}\left(\|\boldsymbol{u}\|_{1}^{2}+\|\boldsymbol{\sigma}\|^{2}\right)
$$

and, using (3.10),

$$
\begin{aligned}
J(\boldsymbol{u}, p, \boldsymbol{\sigma} ; \mathbf{0}, \mathbf{0}) & \geq \Psi / 2-C \lambda^{2} M^{2}\left(\|\boldsymbol{u}\|_{1}^{2}+\|\boldsymbol{\sigma}\|^{2}\right) \\
& \geq C\left(\|(\boldsymbol{u}, p, \boldsymbol{\sigma})\|^{2}-\lambda^{2} M^{2}\left(\|\boldsymbol{u}\|_{1}^{2}+\|\boldsymbol{\sigma}\|^{2}\right)\right) \geq c_{1}\|(\boldsymbol{u}, p, \boldsymbol{\sigma})\|^{2},
\end{aligned}
$$

where $c_{1}>0$ for $\lambda$ and $M$ chosen sufficiently small. This completes the proof.

However the least squares functional $J(\boldsymbol{u}, p, \sigma ; \hat{\boldsymbol{f}}, \boldsymbol{F})$ is not practical. The negative order Sobolev norm $\|\cdot\|_{-1}$ leads to difficulties in the assembly of the linear algebraic equations. In [7], Bochev and Gunzburger used the weighted norm $h\|\cdot\|$ where $h$ denotes some parameter of the finite element space instead of the norm $\|\cdot\|_{-1}$. Hence we will consider the mesh dependent functional in which the residuals of each equation in $L^{2}$ -norm are multiplied by proper mesh dependent weights.

\section{Finite Element Approximations}

We assume that the domain $\Omega$ is a polygon, and $\mathcal{T}^{h}$ is a triangulation of $\Omega$ made of triangular elements $T$ with $h=\max \left\{\operatorname{diam}(T): T \in \mathcal{T}^{h}\right\}$. Thus, the computational domain is given by

$$
\Omega=\left\{\cup T: T \in \mathcal{T}^{h}\right\} .
$$


We assume that the partition $\mathcal{T}^{h}$ is regular and satisfies the inverse assumption. Let $P_{l}(T)$ denote the space of polynomials on $T$ of degree less or equal to $l$. We define the finite element spaces for the approximation of $(\boldsymbol{u}, p, \boldsymbol{\sigma})$ as follows

$$
\begin{gathered}
V^{h}=\left\{\boldsymbol{v}^{h} \in V \cap C(\Omega)^{2}:\left.v^{h}\right|_{T} \in P_{l_{1}+1}(T), \forall T \in \mathcal{T}^{h}\right\} \\
Q^{h}=\left\{q^{h} \in Q \cap C(\Omega):\left.q^{h}\right|_{T} \in P_{l_{2}+1}(T), \forall T \in \mathcal{T}^{h}\right\} \\
\Sigma^{h}=\left\{\tau^{h} \in \Sigma \cap C(\Omega)^{2 \times 2}:\left.\tau^{h}\right|_{T} \in P_{l_{3}+1}(T), \forall T \in \mathcal{T}^{h}\right\} .
\end{gathered}
$$

Let $X^{h}=V^{h} \times Q^{h} \times \Sigma^{h}$ be a finite dimensional subspace of $X$ with the following approximation properties:

$$
\inf _{\varphi^{h} \in \Phi^{h}}\left\|\varphi-\varphi^{h}\right\|_{k} \leq C h^{m}\|\varphi\|_{m+k}, \quad \forall \varphi \in H^{m+k}(\Omega)
$$

where $\Phi^{h}=\left\{\varphi^{h} \in C(\Omega):\left.\varphi^{h}\right|_{T} \in P_{l+1}(T), \forall T \in \mathcal{T}^{h}\right\}$ and $k=0,1$. The space $\Sigma^{h}$ admits the property

$$
\inf _{\tau^{h} \in \Sigma^{h}}\left(\left\|\tau-\tau^{h}\right\|+\left\|\nabla \cdot\left(\tau-\tau^{h}\right)\right\|\right) \leq C h^{m}\left(\|\tau\|_{m}+\|\nabla \cdot \tau\|_{m}\right)
$$

with $\tau \in \Sigma \cap H^{m}(\Omega)$ and $\nabla \cdot \tau \in H^{m}(\Omega)$. The properties hold for finite element spaces consisting of continuous piecewise polynomials based on quasi-uniform triangulations [11] [12].

The mesh dependent least squares functional is defined by the weighted sum in $L^{2}$-norms of the residuals of the equations in (3.3)

$$
\begin{aligned}
J_{h}(\boldsymbol{u}, p, \boldsymbol{\sigma} ; \hat{\boldsymbol{f}}, \boldsymbol{F})= & L h^{-2}\|\nabla \cdot \boldsymbol{u}\|^{2}+h^{-2}\left\|\boldsymbol{\sigma}+\lambda\left(\boldsymbol{u}_{1} \cdot \nabla \boldsymbol{\sigma}+B(\boldsymbol{u}, \boldsymbol{\sigma})\right)-2 \eta \varepsilon(\boldsymbol{u})-F\right\|^{2} \\
& +\left\|\rho\left(\boldsymbol{u} \cdot \nabla \boldsymbol{u}_{1}+\boldsymbol{u}_{1} \cdot \nabla \boldsymbol{u}\right)+\nabla p-\nabla \cdot \boldsymbol{\sigma}-\hat{\boldsymbol{f}}\right\|^{2},
\end{aligned}
$$

where $L$ is a positive constant. The least squares finite element problem is to minimize this functional over $X^{h}$ : seek $\left(\boldsymbol{u}_{h}, p_{h}, \sigma_{h}\right) \in X^{h}$ such that

$$
J_{h}\left(\boldsymbol{u}_{h}, p_{h}, \boldsymbol{\sigma}_{h} ; \hat{\boldsymbol{f}}, \boldsymbol{F}\right)=\inf _{\left(\boldsymbol{v}_{h}, q_{h}, \tau_{h}\right) \in X^{h}} J_{h}\left(\boldsymbol{v}_{h}, q_{h}, \tau_{h} ; \hat{\boldsymbol{f}}, \boldsymbol{F}\right) .
$$

The minimizer of (3.13) necessarily satisfies the Euler-Lagrange equation given by

$$
\mathcal{B}((\boldsymbol{u}, p, \sigma),(\boldsymbol{v}, q, \tau))=\mathcal{F}(\boldsymbol{v}, q, \tau), \quad \forall(\boldsymbol{v}, q, \tau) \in X^{h}
$$

where

$$
\begin{aligned}
& \mathcal{B}((\boldsymbol{u}, p, \boldsymbol{\sigma}),(\boldsymbol{v}, q, \boldsymbol{\tau}))= \int_{\Omega} L h^{-2}(\nabla \cdot \boldsymbol{u})(\nabla \cdot \boldsymbol{v}) \\
&+h^{-2}\left(\boldsymbol{\sigma}+\lambda\left(\boldsymbol{u}_{1} \cdot \nabla \boldsymbol{\sigma}+B(\boldsymbol{u}, \boldsymbol{\sigma})\right)-2 \eta \varepsilon(\boldsymbol{u})\right) \cdot\left(\boldsymbol{\tau}+\lambda\left(\boldsymbol{u}_{1} \cdot \nabla \boldsymbol{\tau}+B(\boldsymbol{v}, \boldsymbol{\tau})\right)-2 \eta \varepsilon(\boldsymbol{v})\right) \\
&+\left(\rho\left(\boldsymbol{u} \cdot \nabla \mathbf{u}_{1}+\boldsymbol{u}_{1} \cdot \nabla \boldsymbol{u}\right)+\nabla p-\nabla \cdot \boldsymbol{\sigma}\right) \cdot\left(\rho\left(\boldsymbol{v} \cdot \nabla \boldsymbol{u}_{1}+\boldsymbol{u}_{1} \cdot \nabla \boldsymbol{v}\right)+\nabla q-\nabla \cdot \tau\right) \mathrm{d} x, \\
& \mathcal{F}(\boldsymbol{v}, q, \boldsymbol{\tau})=\int_{\Omega} h^{-2} \boldsymbol{F}:\left(\tau+\lambda\left(\boldsymbol{u}_{1} \cdot \nabla \tau+B(\boldsymbol{v}, \boldsymbol{\tau})\right)-2 \eta \varepsilon(\boldsymbol{v})\right)+\hat{\boldsymbol{f}} \cdot\left(\rho\left(\boldsymbol{v} \cdot \nabla \boldsymbol{u}_{1}+\boldsymbol{u}_{1} \cdot \nabla \boldsymbol{v}\right)+\nabla q-\nabla \cdot \tau\right) \mathrm{d} x
\end{aligned}
$$

and the double-dot product is defined as

$$
\sigma: \tau=\sum_{i, j} \sigma_{i j} \tau_{i j} .
$$

Based on Theorem 1, we establish the ellipticity of the functional $J_{h}$ in Theorem 2.

Theorem 2. For any $(\boldsymbol{u}, p, \sigma) \in X^{h}$, there exist positive constants $c_{1}$ and $c_{2}$, which depend on $\lambda, \eta$ and $\rho$, such that

$$
c_{1}\|(\boldsymbol{u}, p, \boldsymbol{\sigma})\|^{2} \leq J_{h}(\boldsymbol{u}, p, \boldsymbol{\sigma} ; \mathbf{0}, \mathbf{0}) \leq c_{2} h^{-2}\|(\boldsymbol{u}, p, \boldsymbol{\sigma})\|^{2},
$$

for $h<1$. 
Proof. The first inequality in (3.15) is straightforward from Theorem 1. To prove the upper bound, we assume that the spaces $Q^{h}$ and $\Sigma^{h}$ satisfy the following inverse inequalities

$$
\|\nabla p\| \leq C h^{-1}\|p\|
$$

and

$$
\|\nabla \cdot \sigma\| \leq C h^{-1}\|\sigma\|
$$

From the triangle inequality, we obtain

$$
\begin{aligned}
& \left.J_{h}(\boldsymbol{u}, p, \boldsymbol{\sigma} ; \mathbf{0 , 0})\right] \\
& =L h^{-2}\|\nabla \cdot \boldsymbol{u}\|^{2}+h^{-2}\left\|\boldsymbol{\sigma}+\lambda\left(\boldsymbol{u}_{1} \cdot \nabla \boldsymbol{\sigma}+B(\boldsymbol{u}, \boldsymbol{\sigma})\right)-2 \eta \varepsilon(\boldsymbol{u})\right\|^{2}+\left\|\rho\left(\boldsymbol{u} \cdot \nabla \boldsymbol{u}_{1}+\boldsymbol{u}_{1} \cdot \nabla \boldsymbol{u}\right)+\nabla p-\nabla \cdot \boldsymbol{\sigma}\right\|^{2} \\
& \leq L h^{-2}\|\boldsymbol{u}\|_{1}^{2}+C h^{-2}\left(\|\boldsymbol{\sigma}\|^{2}+\lambda^{2}\left\|\boldsymbol{u}_{1} \cdot \nabla \boldsymbol{\sigma}\right\|^{2}+\|\boldsymbol{u}\|_{1}^{2}+\|p\|^{2}+\|\boldsymbol{\sigma}\|^{2}\right)+\rho^{2}\|\boldsymbol{u}\|_{1}^{2} \\
& \leq c_{2} h^{-2}\left(\|\boldsymbol{u}\|_{1}^{2}+\|\boldsymbol{\sigma}\|^{2}+\|p\|^{2}+\left\|\boldsymbol{u}_{1} \cdot \nabla \boldsymbol{\sigma}\right\|^{2}\right) .
\end{aligned}
$$

This completes the proof of the theorem.

By virtue of Theorem 2 and the Lax-Milgram theorem, we establish the following theorem.

Theorem 3. For any $h<1$, the functional (3.13) has the unique minimizer out of $X^{h}$, i.e., there exists a unique solution satisfies the Euler-Lagrange equation (3.14).

Now we derive error estimates for the least-squares finite element solution $\left(\boldsymbol{u}^{h}, p^{h}, \boldsymbol{\sigma}^{h}\right)$ which satisfies (3.14).

Theorem 4. Assume that $(\boldsymbol{u}, p, \boldsymbol{\sigma}) \in X$ is the solution to (3.3), then the least-squares finite element solution $\left(\boldsymbol{u}^{h}, p^{h}, \sigma^{h}\right) \in X^{h}$ satisfies

$$
\left\|\left(\boldsymbol{u}-\boldsymbol{u}^{h}, p-p^{h}, \boldsymbol{\sigma}-\boldsymbol{\sigma}^{h}\right)\right\| \leq C h^{m-1}\left(\|\boldsymbol{u}\|_{m+1}+\|p\|_{m}+\|\boldsymbol{\sigma}\|_{m}+\|\nabla \cdot \boldsymbol{\sigma}\|_{m}\right),
$$

for $m \leq l+1$.

Proof. From Theorem 2, we obtain the following bound

$$
\left\|\left(\boldsymbol{u}-\boldsymbol{u}^{h}, p-p^{h}, \boldsymbol{\sigma}-\boldsymbol{\sigma}^{h}\right)\right\| \leq \inf _{\left(\boldsymbol{v}_{h}, q_{h}, \tau_{h}\right) \in X^{h}} \frac{c_{2}}{c_{1}} h^{-1}\left\|\left(\boldsymbol{u}-\boldsymbol{v}^{h}, p-q^{h}, \boldsymbol{\sigma}-\tau^{h}\right)\right\| .
$$

Combining the properties (3.11) and (3.12), we have

$$
\begin{aligned}
\left\|\left(\boldsymbol{u}-\boldsymbol{u}^{h}, p-p^{h}, \boldsymbol{\sigma}-\boldsymbol{\sigma}^{h}\right)\right\| & \leq C h^{-1}\left(\left\|\boldsymbol{u}-\boldsymbol{v}^{h}\right\|_{1}+\left\|p-q^{h}\right\|+\left\|\sigma-\tau^{h}\right\|+\left\|\boldsymbol{u}_{1} \cdot \nabla\left(\sigma-\tau^{h}\right)\right\|\right) \\
& \leq C h^{m-1}\left(\|\boldsymbol{u}\|_{m+1}+\|p\|_{m}+\|\sigma\|_{m}+\|\nabla \cdot \sigma\|_{m}\right),
\end{aligned}
$$

This completes the proof of the theorem.

\section{Conclusion}

In this paper, we have proposed and analyzed a weighted least-squares method for the approximate solution of the upper-convected Maxwell fluid. The weights in our least-squares functional involve mesh dependent weight and mass conservation constant. The homogenous functional is shown to be equivalent to a natural norm. A prior error estimate is given for the finite element solutions. An adaptive least-squares finite element method for this viscoelastic fluid model will be discussed in the future.

\section{Acknowledgements}

The authors' work is supported by the National Science Foundation of China (No. 11271247) and the Natural Science Foundation of Hebei Province (No. G2013402063).

\section{References}

[1] Chen, T.F., Lee, H. and Liu, C.C. (2013) Numerical Approximation of the Oldroyd-B Model by the Weighted Least- 
Squares/Discontinuous Galerkin Method. Numerical Methods for Partial Differential Equations, 29, 531-548. http://dx.doi.org/10.1002/num.21719

[2] Cai, Z. and Ku, J. (2006) The $L^{2}$ Norm Error Estimates for the Div Least-Squares method. SIAM Journal on Numerical Analysis, 44, 1721-1734. http://dx.doi.org/10.1137/050636504

[3] Zhou, S.L. and Hou, L. (2015) Decoupled Algorithm for Solving Phan-Thien-Tanner Viscoelastic Fluid by Finite Element Method. Computer \& Mathematics with Applications, 69, 423-437. http://dx.doi.org/10.1016/j.camwa.2015.01.006

[4] Cai, Z., Lazarov, R. and Manteuffel, T.A. and McCormick, S.F. (1994) First-Order System Least Squares for SecondOrder Partial Differential Equations: Part I. SIAM Journal on Numerical Analysis, 31, 1785-1799. http://dx.doi.org/10.1137/0731091

[5] Cai, Z., Lee, B. and Wang, P. (2004) Least-Squares Methods for Incompressible Newtonian Fluid Flow: Linear Stationary Problems. SIAM Journal on Numerical Analysis, 42, 843-859. http://dx.doi.org/10.1137/S0036142903422673

[6] Lee, H.C. and Chen, T.F. (2015) Adaptive Least-Squares Finite Element Approximations to Stokes Equations. Journal of Computational and Applied Mathematics, 280, 396-412. http://dx.doi.org/10.1016/j.cam.2014.11.041

[7] Bochev, P.B. and Gunzburger, M.D. (1995) Least-Squares Methods for the Velocity-Pressure-Stress Formulation of the Stokes Equations. Computer Methods in Applied Mechanics and Engineering, 126, 267-287. http://dx.doi.org/10.1016/0045-7825(95)00826-M

[8] Lee, H.C. (2014) An Adaptively Refined Least-Squares Finite Element Method for Generalized Newtonian Fluid Flows Using the Carreau Model. SIAM Journal on Scientific Computing, 36, A193-A218. http://dx.doi.org/10.1137/130912682

[9] Fan, Y., Tanner, R.I. and Phan-Thien, N. (1999) Galerkin/Least-Square Finite-Element Methods for Steady Viscoelastic Flows. Journal of Non-Newtonian Fluid Mechanics, 84, 233-256. http://dx.doi.org/10.1016/S0377-0257(98)00154-2

[10] Cai, Z., Manteuffel, T.A. and McCormich, S.F. (1995) First-Order System Least Squares for Velocity-Vorticity-Pressure from of the Stokes Equations, with Application to Linear Elasticity. Electronic Transactions on Numerical Analysis, 3, 150-159.

[11] Cai, Z. and Westphal, C.R. (2009) An Adaptive Mixed Least-Squares Finite Element Method for Viscoelastic Fluids of Oldroyd Type. Journal of Non-Newtonian Fluid Mechanics, 159, 72-80. http://dx.doi.org/10.1016/j.jnnfm.2009.02.004

[12] Braess, D. (2007) Finite Elements: Theory, Fast Solvers, and Applications in Solid Mechanics. Cambridge University Press, Cambridge. http://dx.doi.org/10.1017/CBO9780511618635 